\title{
Cerebellar abscess secondary to extradural internal protuberance epidermal cyst
}

\section{Marleen,}

\section{Syafrizal Abubakar,}

Achmad Jana Maulana,

Michael Norman Jusman

Department of Neurosurgery,

Tangerang Public Hospital,

Tangerang, Indonesia

\section{Cite this article:}

Marleen, Abubakar S, Maulana AJ, Jusman MN. Cerebellar abscess secondary to extradural internal protuberance epidermal cyst. Neurologico Spinale Medico Chirurgico.2019;2(2): 37-40. DOI: I 0.36444/nsmc.v2i2.4

Corresponding author:

Marleen

Department of Neurosurgery, Tangerang

Public Hospital, Indonesia

marleenlee1@gmail.com

\begin{abstract}
An Intracranial epidermoid cyst is a rare congenital anomaly developed because of dysembryoplasia during the third to fifth week of gestation. We describe the unusual epidermal cyst which originating extradurally in internal protuberance in which causes cerebellar brain abscess. Neurosurgical intervention is advocated to prevent the ongoing risk of significant complications. Meticulous care was taken to grossly excised all the pathologic tissue to avoid recurrence.
\end{abstract}

Keywords: Epidermal cyst, Cerebellar Abscess, Aseptic Meningitis, Dermal Sinus

\section{Introduction}

An Intracranial epidermoid cyst is a rare congenital anomaly developed because of dysembryoplasia during the third to fifth week of gestation. ${ }^{1}$ During this time the medullary tube is being formed from the neural groove and the ectoblast is committed to either a neural or cutaneous lineage. ${ }^{2}$ These trapped committed cutaneous cells may then give rise to intraduraldermoids and epidermoids. If there is persistence of the cutaneous tract with overlying skin, a communicating dermal sinus may develop. ${ }^{3}$ Histologically they are composed of an outer capsule, an epithelial layer and in some cases an inner cystic fluid. The outer layer is composed of connective tissue, which surrounds a layer of keratinized stratified squamous epithelium. As the epithelial layer desquamates, the cells accumulate and form a cholesterol-rich inner layer that gives the cyst its characteristic pearly white appearance. ${ }^{4}$ Epidermoid tumors are also called as inclusion tumors because they rise from remnants of epithelial tissue during the neural tube closure at the third and fifth week of developmental stages of a fetus. These account for $0.2 \%$ to $1.8 \%$ of all intracranial tumors. ${ }^{5}$ Epidermoid cyst was first discovered by an artist in a French medical school in $1807 .{ }^{6}$ Cruveilhier described the epidermoids as "pearly tumors" and Dandy as "most beautiful tumors of the body". Among epidermoids, the highest incidence of the housing of these lesions was at cerebellopontine angle which were $51.8 \%$, followed by lateral ventricle with $4.9 \%$, fourth ventricle with $3.2 \%$, third ventricle with $0.7 \%$ and other sites includes $39.4 \% .^{7}$ The treatment is generally carrying a very good prognosis for epidermoid cyst is the total surgical resection grossly. ${ }^{8}$ Whereas the decompression of cyst can just be a temporary relief, ${ }^{9-11}$ while subtotal resection can result in recurrence of tumor which is estimated to appear at the time of an interval equal to the age of patients with the addition of nine months to the initial presentation of symptoms. ${ }^{12}$

The main post-operative complication is aseptic meningitis. ${ }^{13,14}$ Aseptic meningitis stands between $2-50 \%$ incidences occurring postoperatively according to literature data; patients mostly whose cysting capsule has been excised incompletely prone to this complication. ${ }^{15,16}$ Chemical meningitis results in the rupture of the cyst. Removal of epidermoid cyst leads to complications such as hydrocephalus, which may be obstructive or communicating. ${ }^{17}$ The surgical resection of epidermoid in the posterior fossa may sometimes lead to hypertrophic olivary degeneration. The 
clinical picture of this includes palatal myoclonus, which is an involuntary movement of oropharynx, uvula and soft palate, ocular myoclonus and dentatorubral tremor. ${ }^{18}$

As the growth rate of these epidermoid tumors resembles the same as the human skin any cell retained can lead to the risk of recurrence or regeneration. According to the recently published studies, subtotal excision of posterior fossa epidermoid cyst has the recurrence rate between $0-54 \% .{ }^{19-21}$ Subtotal removal of the fourth ventricular epidermoid cyst carries a good prognosis, ${ }^{22}$ even if the tumor is subtotally resected long term survival can be expected in atypical epidermoid cystic patients. Even if the tumor is excised subtotally in neurologically preserved patients the prognosis remains quite good which is confirmed by the experience..$^{23,24}$ The location of the tumor and the time taken from onset to diagnosis are the important factors for the prognosis than the size of the tumor.

\section{Case Report}

A 4-year-old child presented with nausea and vomitus, ataxia and vertigo. He also has blurry vision since last week before admission. He previously had a history of soft tissue lump which evident of purulent discharge and chronically healed. This lump was noticed since he was born. There was no evidence of fever or other sources of infection.

Physical examination showed cerebellar signs including disdiadokinesis, intention tremor, and ataxia. Mild papil edema on both eyes without any evidence of false localizing sign. We did not find any soft tissue mass, but we found residual punctum in the skin overlying external occipital protuberance.

We initially diagnosed and managed the patient with posterior fossa brain abscess with unknown origin, with posterior fossa tumor as a differential diagnosis. We decide to elect posterior fossa craniotomy for cerebellar abscess drainage for obtaining the purulent sample for culture or diagnostic purposes and for posterior fossa decompression

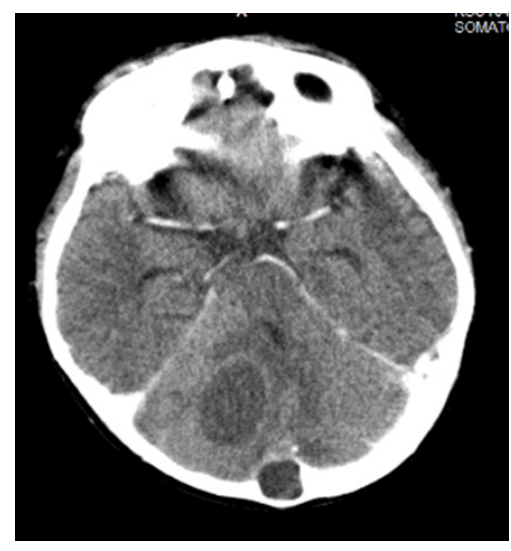

Fig 1. Non-Contrast Head CT Scan showed rounded bony erosion in internal protuberance with adjacent cystic hypodense lesion in the right cerebellar hemisphere, distorting the fourth ventricle.
In prone position under general anesthesia, the head was fixed by three pinhead fixation (DoroTM) in midline fashioned and avoid jugular drainage obstruction. Midline suboccipital linear skin incision was made, and we extend $3-4 \mathrm{~cm}$ above inion to achieve adequate exposure External Occipital Protuberance. Midline avascular skin incision was made to avoid excessive bleeding from suboccipital muscle tissue. Burrhole and suboccipital craniotomy was made with exposure of end part of superior sagittal sinus, torcular herophili, and bilateral transverse sinus. Pearl liked mass was found just beneath the confluence which produced white purulent discharge. We excised the tissue stalk and found hairy and subcutaneous fat-liked tissue beneath the mass, occupying the remaining occipital sinus. Occipital sinus was ligated, and confluence was preserved to avoid profuse bleeding and venous infarction. After durotomy was made, the right cerebellar hemisphere looks yellowish and we made $1.5 \mathrm{~cm}$ corticotomy on superior lobule of cerebellar hemisphere and drainage all purulent containing abscess. We also obtained granulating tissue inside the abscess capsule and we irrigate the abscess cavity with normal saline until there was no evidence of purulent material inside the cavity. Since we excised the pathologic posterior fossa dura, we used dural substitute (duragenTM) for duraplasty. Osteoplastic autologous cranioplasty was made because we believe that there is no pathologic involvement in patient bony erosion. All the muscular and fascial layers were closed layer by layer in watertight fashioned to avoid pseudomeningocele after surgery. The patient was extubated the day after surgery and discharge from intensive care with single antibiotic coverage (Ceftriaxone) and additional steroid for attenuating post-surgical edema.
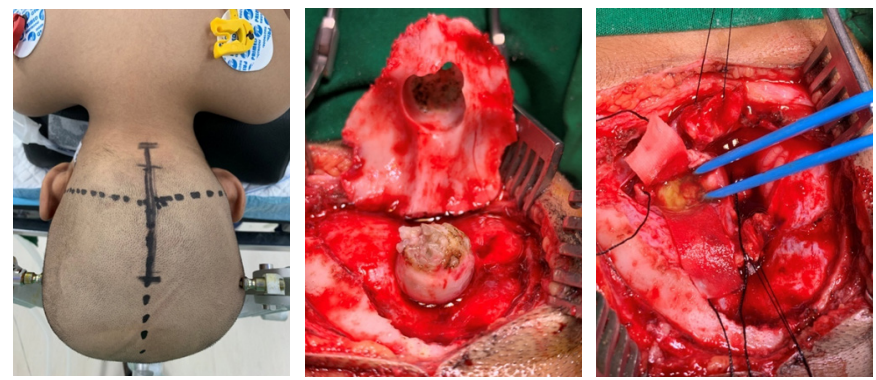

Fig 2. Left picture shows patient in prone position, linear midline suboccipital skin incision was made with 3-4 cm superior extension to exposed external occipital protuberance adequately. The middle picture shows the pearl-like mass with overlying internal occipital protuberance erosion was revealed after craniotomy was made. The mass occupies just beneath the confluence. And the right picture shows the Intracavitary purulent content was revealed after $1.5 \mathrm{~cm}$ corticotomy on the right superior lobule.

The patient developed fever until 4 days post operatively. We then added another antibiotics (Metronidazole) to coverage the anaerobic microbial infections possibilities on the day 3 follow up. The fever showed improvement with antipyretics and we assumed that it was caused by aseptic meningitis. The patient showed improve cerebellar symptoms and visual acuity, and discharge on day 6 after surgery. 


\section{Discussion}

Extradural Intracranial epidermoid cysts were previously reported from several authors. Liu et al., ${ }^{7}$ report a case in which extradural intracranial epidermoid cyst caused supratentorial brain abscess. Brain Abscess pathogenesis were assumed to develop because of the direct connection between cyst and overlying skin via a dermal sinus. This patient had a history of purulent discharge on his occipital region, where we identified punctum like appearance on his occipital skin. Lack of awareness or occult nature history of the disease leads the epidermal cyst into cerebellar abscess development.

The child became unsteady while walking and started to intermittently vomiting and vertigo. Since physical examination warrants a suspicious cerebellar pathology, Contrast-Enhanced CT Scan was undertaken to ruled out any pathology in cerebellar anatomy. We found bony erosion on internal occipital protuberance and also thin-walled enhancement cystic lesion on the right cerebellar hemisphere.

Midline Suboccipital Craniotomy showed a pearl-like mass beneath the external occipital protuberance which has a broad and thickened attachment with underlying midline dura. Corticotomy was made and we drainage purulent content of abscess cavity and removal all granulated tissue content. Since there were no sufficient dural tissue to approximate, we use dural substitute to help dural closure at the end of the procedure.

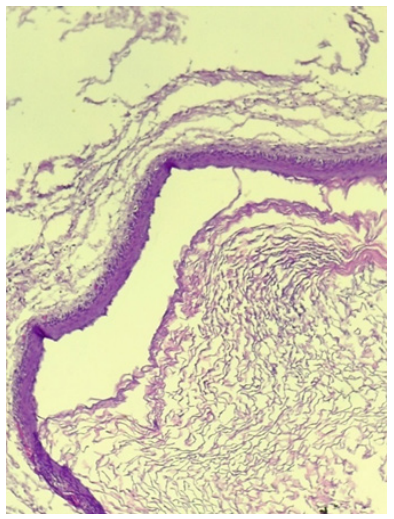

Fig 3. Epidermoid cyst lined by benign-appearing squamous epithelium and filled with keratinous material.

\section{Conclusion}

We have presented a quite rare case with extradural epidermoid cyst and associated intracerebellar abscess. Early neurosurgical intervention is advocated to prevent the ongoing risk of serious complications. Meticulous tumor resection plays an important step to avoid recurrence and improve neurological symptoms. Clarifying the pathogenesis would lead to effective preventive measures.

\section{Acknowledgment}

We appreciate the great help from our Pathology Department, dr. Ratna for providing pathological images and expertise.

\section{References}

1. Reddy MP, Song J, Hong X, Ma Z. Intracranial epidermoid cyst: characteristics, appearance, diagnosis, treatment and prognosis. SciLett. 2015; 3(3):102-110.

2. Tampieri D, Melanson D, Ethier R. MR imaging of epidermoid cysts. AJNR. 1989; 10:351-56.

3. Sachs E, Horrax G. A cervical and lumbar pilonidal sinus communicating with intraspinaldermoids. Report of 2 cases and review of the literature. J Neurosurg. 1949; 6:97-112. doi: 10.3171/jns.1949.6.2.0097.

4. Uchino A, Hasuo K, Matsumoto, et al. Intracranial epidermoid carcinoma: CT and MRI. Neuroradiology. 1995; 37;155-58.

5. Chandler WF, Farhat SM, Pauli FJ. Intrathalamicepidermoid tumor. Case report. J neurosurg. 1975; 43:614-617. doi: 10.3171/jns.1975.43.5.0614.

6. Grant FC, Austin GM. Epidermoids: clinical evaluation and surgical results. Neurosurg. 1950;7:190-198. doi: 10.3171/jns.1950.7.3.0190.

7. Liu YG. Epidermoid cyst. In: Liu YG, (Ed). Congenital cranial-cerebral diseases. 1st ed. Jinan: Jinan Press. P. 92-96, 1993.

8. Nakao Y, Nonaka S, Yamamoto T, et al. Malignant transformation 20 years after partial removal of intracranial epidermoid cyst. Neurologia Medico-Chirurgica. 2010;50(3)236-239. doi: 10.2176/nmc.50.236.

9. Fiume D, Gazzeri G, Spallone A, et al. Epidermoid cysts of the fourth ventricle. SurgNeurol. 1988;29:178-182. doi: https:/doi. org/10.1016/0090-3019(88)90002-X

10. Tancredi A, Fiume D, Gazzeri G. Epidermoid cysts of the fourth ventricle: very long follow up in 9 cases and review of the literature. ActaNeurochir. 2003;145:905-910. doi: 10.1007/s00701-003-0008-3.

11. Zhou LF. Intracranial epidermoidtumours: Thirty-seven years of diagnosis and treatment. Br J Neurosurg. 1990; 4:211-216.

12. Ziyal IM, Bilginer B, Bozkurt G, et al. Epidermoid cyst of brain stem symptomatic in childhood. Childs NervSyst. 2005; 12:21:1025-1029. doi: 10.1007/s00381-005-1172-y.

13. Bret P, Remond J, Fischer C, et al. Epidermoid cyst of the fourth ventricle. Br J Neurosurg. 1988; 2:109-113. doi: 10.3109/02688698808999667.

14. Faught RE, Chandra-Sekar B, Bonnin JM, et al. Medical pathology conference. A 34-year-old man with recent onset of ataxia. Ala J Med Sci. 1986; 23:408-413.

15. Samii M, Tatagiba M, Piquer J, et al. Surgical treatment of epidermoid cysts of the cerebellopontine angle. J Neurosurg. 1996;84:14-19. doi: 10.3171/jns.1996.84.1.0014.

16. Zerah M, Emery E, Comoy J, et al. Epidermoid cyst of the 4th ventricle. Apropos of a case in a child and review of the literature. Neurochirurgia. 1993; 39(4):241-247.

17. Akar Z, Tanriover N, Tuzgen S, et al. Surgical treatment of intracranial epidermoid tumors. Neurol Med Chir. 2003; 43(6):275-281. doi: 10.2176/nmc.43.275.

18. Pearce JM. Palatal Myoclonus (syn. Palatal tremor). EurNeurol. 2008; 60:312-315. doi: 10.1159/000159929.

19. Mohanty A, Venkatrama SK, Rao BR, et al. Experience with cerebellopontine angle epidermoids. Neurosurg. 1997; 40:24-30.

20. Lunardi P, Missori P, Innocenzi G, et al. Long term results of surgical treatment of cerebellopontineangleepidermoids. ActaNeurochir. 1990;103105-108.

21. De Souza CE, De Souza R, Da Costa S, et al. Cerebellopontine angle epidermoid cysts: a report on 30 cases. J NeurolNeursurg Psychiatry. 1989; 52:986-990. doi: 10.1136/jnnp.52.8.986.

22. Tancredi A, Fiume D, Gazzeri G. Epidermoid cyst of fourth ventricle: A very long follow up in 9 cases and review of literature. Acta Neurochir 
(Wien). 2003; 145:905-911. doi: 10.1007/s00701-003-0008-3.

23. Altzschuler EM, Jungreis CA, Sekhar LN, et al. Operative treatment of intracranial epidermoid cyst and cholesterol granulomas: report of 21 cases. Neurosurg. 1990;26:606-614. doi: 10.1097/00006123-
199004000-00008.

24. Berger MS, Wilson CB. Epidermoid cyst of the posterior fossa. Neurosurg. 1985; 62:214-219. doi: 10.3171/jns.1985.62.2.0214. 